\title{
Restless-Legs-Syndrom
}

\section{Auch Eisenmangel lässt die Beine zappeln}

— Eisenmangel zählt zu den häufigsten Mineralstoffdefiziten, wird jedoch aufgrund seiner unspezifischen Symptomatik oft nicht erkannt. Neben den typischen sehr allgemeinen Beschwerden wie Müdigkeit, Konzentrationsstörungen, Leistungsabfall und Rhagaden scheint Eisenmangel auch beim Restless-Legs-Syndrom eine Schlüsselrolle zu spielen.

Der Gesamteisengehalt des Organismus eines gesunden Erwachsenen beträgt ungefähr $3,5-5 \mathrm{~g}$. Davon sind etwa 65-70\% im Hämoglobin enthalten, etwa $4 \%$ im sog. Funktionseisen (Myoglobin, eisenhaltige Enzyme) und etwa 25\% im sog. Depoteisen (Ferritin, Hämosiderin). Die täglichen Eisenverluste betragen beim Mann ca. $1 \mathrm{mg}$ und bei der Frau im gebärfähigen Alter ca. 2 mg.

Mit der täglichen Nahrung werden ca. 10-15 mg Eisen zugeführt, wovon nur 10-15\% resorbiert werden. Das Nahrungseisen kann daher höchstens physiologische Eisendefizite ausgleichen, jedoch keinesfalls einen erhöhten Eisenbedarf.
Aus einer lang anhaltenden Eisenunterversorgung kann sich ohne Substitution leicht eine chronische Eisenmangelanämie entwickeln.

Die medikamentöse Substitution von oralem Eisen sollte über mindestens zwölf Wochen erfolgen. Die WHO empfiehlt dafür Eisensalze mit 60-120 mg zweiwertigem Eisen und verzögerter Freisetzung. Zur Auffüllung der Eisenspeicher ist da-

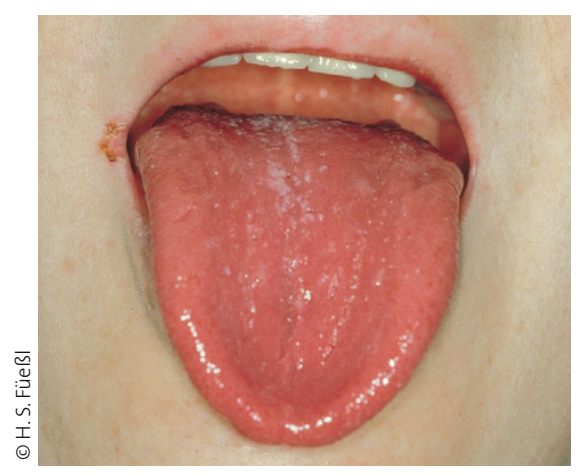

Sichtbare Eisenmangelanämie: glatte rote Zunge mit Atrophie der filiformen Papillen und Mundwinkelrhagaden. nach eine Gabe über weitere drei Monate ratsam. Dabei sollte die Eisensubstitution bevorzugt morgens auf nüchternen Magen erfolgen. Die Kombination mit Vitamin C steigert die Bioverfügbarkeit, sodass Eisenpräparate am besten mit Orangensaft eingenommen werden. Für eine gute Compliance ist eine gute Verträglichkeit des Eisenpräparats wichtig.

Ein Präparat, das diese Anforderungen erfüllt, ist Tardyferon ${ }^{\circledR}$. Mit seiner speziellen Galenik passt sich die Resorption an die Bedingungen bei Eisenmangel an: Nach sechs Stunden sind 90\% des Eisens freigesetzt, davon können $70 \%$ im Duodenum und weitere $15 \%$ im Jejunum resorbiert werden. Das Eisen steht in den einzelnen Darmabschnitten gemäß der resorptiven Kapazität zur Verfügung. Es kommt also nicht zu einem massiven Überangebot an Eisen und daher zu deutlich weniger Irritationen der Darmschleimhaut.

- Dr. med. Kirsten Westphal
Ouelle: Pierre Fabre Pharma

\section{Alzheimerkrankheit}

\section{Antidementive Therapie mit Langzeitwirkung}

- Die Wirksamkeit des Antidementivums Memantine (z.B. Axura ${ }^{\circledR}$ ) auf die Kerndomänen der Alzheimerdemenz Kognition, alltagspraktische Fähigkeiten, soziales Verhalten und klinischer Gesamteindruck - ist durch randomisierte Doppelblindstudien und Metaanalysen mehrfach belegt. Dass diese Effekte nicht in kürzester Zeit „verpuffen“, sondern langfristig erhalten bleiben und mit der Dauer der Therapie sogar deutlicher werden, zeigt eine prospektive Longitudinalstudie der Harvard Medical School.

Die Forscher hatten die im klinischen Alltag erfassten Daten von 382 Patienten mit Alzheimerdemenz über einen durchschnittlichen Beobachtungszeitraum von 30 Monaten analysiert. Die Patienten waren entweder mit Memantine kombiniert mit einem Acetylcholinesterasehemmer (AChEH) $(n=116)$, einem AChEH allein ( $n$ = 122) oder einer Standardtherapie ohne Antidementivum behandelt worden. Es zeigte sich, dass unter Memantine plus AChEH der Verlust sowohl von kognitiven Leistungen (gemäß Blessed Dementia Scale) als auch von alltagspraktischen Fähigkeiten (gemäß Activities of Daily Living-Skala) signifikant langsamer erfolgte als mit den beiden anderen Therapien. Dabei nahm die relative Effektgröße mit der Dauer der Memantinetherapie kontinuierlich und signifikant zu.

\section{Messbarer Nutzen auch} unter Alltagsbedingungen

Der Nutzen einer Memantinetherapie bei Alzheimerpatienten tritt auch unter All- tagsbedingungen klar zutage. In zwei prospektiven, nicht interventionellen Studien bei Patienten im häuslichen Umfeld ( $n=2485)$ und bei Pflegeheimpatienten $(n=1325)$ gelang es, die kognitiven Funktionen im MMSE-Test bei bis zu 80\% der Patienten gegenüber dem Ausgangswert zu verbessern bzw. zu stabilisieren.

Der auch in den Leitlinien empfohlene NMDA-Antagonist Memantine ist bei Patienten mit moderater bis schwerer Alzheimerdemenz indiziert. Die einmal tägliche Gabe der 20-mg-Tablette und ihre gute Verträglichkeit erleichtern die Therapie bei multimorbiden Demenzpatienten.

- BS

Quelle: Satellitensymposium, DGPPNKongress, Berlin, 25. November 2009 (Veranstalter: Merz) 